\title{
Walks, partitions, and normal ordering
}

\author{
Askar Dzhumadil'daev Damir Yeliussizov \\ Kazakh-British Technical University \\ Almaty, Kazakhstan \\ dzhuma@hotmail.com yeldamir@gmail.com
}

Submitted: Apr 11, 2015; Accepted: Sep 30, 2015; Published: Oct 16, 2015

Mathematics Subject Classifications: 05A18, 05A30, 11B73

\begin{abstract}
We describe the relation between graph decompositions into walks and the normal ordering of differential operators in the $n$-th Weyl algebra. Under several specifications, we study new types of restricted set partitions, and a generalization of Stirling numbers, which we call the $\lambda$-Stirling numbers.
\end{abstract}

Keywords: Decompositions of graphs; differential operators; Weyl algebra; set partitions; Stirling numbers

\section{Introduction}

Let $G=(V, E)$ be a digraph with an ordered set of edges $E=\left(e_{1}, \ldots, e_{m}\right)$. A walk of $G$ is any sequence of edges $e_{i_{1}} \ldots e_{i_{\ell}}$ such that the terminal vertex of $e_{i_{k}}$ coincides with the initial vertex of $e_{i_{k+1}}$ for all $k<\ell$. The walk $e_{i_{1}} \ldots e_{i_{\ell}}$ is called increasing if $i_{1}<\cdots<i_{\ell}$.

Consider decompositions of $G$ into edge-disjoint increasing walks. This setting generalizes set partitions, because when $G$ has only one vertex and $m$ labeled loops $(1,1)$, decompositions into increasing walks correspond to partitions of the set $[m]:=\{1, \ldots, m\}$ into subsets. If $G$ is decomposed into one walk (with distinct edges), it is an Euler tour. If $G$ is a path and edges along the path are labeled by an arbitrary permutation $\sigma \in S_{m}$, decompositions into minimal number of increasing walks index the descent set of $\sigma$.

This interpretation arises from the normal ordering problem in the Weyl algebra. The $n$-th Weyl algebra $A_{n}$ is an associative algebra with $2 n$ generators $x_{1}, \ldots, x_{n}, \partial_{1}, \ldots, \partial_{n}$ subject to relations $\left[\partial_{i}, x_{j}\right]=\delta_{i, j},\left[x_{i}, x_{j}\right]=0,\left[\partial_{i}, \partial_{j}\right]=0$, where $[a, b]=a b-b a$ is the commutator and $\delta_{i, j}$ is the Kronecker delta. The element $w \in A_{n}$ is normally ordered if it is expressed in the form $w=\sum_{k} c_{k} \prod_{i} x_{i} \prod_{j} \partial_{j}$. For the digraph $G$, the special case of our normal ordering formula is

$$
\prod_{\ell=1}^{\vec{m}} x_{i_{\ell}} \partial_{j_{\ell}}=\sum_{\begin{array}{c}
\text { decompositions into increasing } \\
\text { walks with sources } I
\end{array}} \prod_{i \in I} x_{j} \prod_{j \in J} \partial_{j},
$$




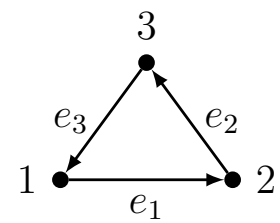

Figure 1: A digraph with an ordered set edges.

where $\left(i_{\ell}, j_{\ell}\right)$ is an $\ell$-th edge of $G$ and $J$ is the corresponding multiset of sinks (which is determined uniquely from the given sources $I$ ). For example, the graph in Figure 1 has four decompositions into increasing walks:

$$
e_{1} e_{2} e_{3}, \quad e_{1} e_{2} \cup e_{3}, \quad e_{1} \cup e_{2} e_{3}, \quad e_{1} \cup e_{2} \cup e_{3} .
$$

This corresponds to the normal ordering

$$
x_{1} \partial_{2} x_{2} \partial_{3} x_{3} \partial_{1}=x_{1} \partial_{1}+x_{1} x_{3} \partial_{3} \partial_{1}+x_{1} x_{2} \partial_{2} \partial_{1}+x_{1} x_{2} x_{3} \partial_{2} \partial_{3} \partial_{1},
$$

where the sources and sinks of walks are exactly the indices of terms. In fact, this formula is a graph-theoretic version of Olshanski's analog of Wick's formula [26] (cf. $[27])$. Collecting the terms we can rewrite

$$
\prod_{\ell=1}^{\vec{m}} x_{i_{\ell}} \partial_{j_{\ell}}=\sum_{I} S_{G}(I) \prod_{i \in I} x_{j} \prod_{j \in J} \partial_{j},
$$

where $S_{G}(I)$ is the number of decompositions of $G$ into increasing walks with multiset of sources $I$. In the case of the first Weyl algebra $A_{1}$ this formula gives the well-known expansion

$$
(x \partial)^{m}=\sum_{i} S(m, i) x^{i} \partial^{i}
$$

for Stirling numbers of the second kind $S(m, i)$.

We introduced this combinatorial model in a more general setting in [15], where we studied algebraic applications, polynomial identities and commutators on Weyl algebra. In this paper we focus on combinatorial aspects of walk decompositions and various specifications of the normal ordering interpretations, such as restricted set partitions and generalized Stirling numbers. We study walk decompositions and the $G$-Stirling functions which enumerate decompositions by sources of walks. As we see, the values of the $G$-Stirling function serve as connection constants in the normal ordering problem.

The setting of decomposing graphs into walks is a source for certain types of restricted set partitions. For example, assume that digraph has 2 vertices and edges $e_{1}, e_{3}, \ldots$ going from vertex 1 to 2 and the remaining edges $e_{2}, e_{4}, \ldots$ going back from 2 to 1 . Decompositions on this model generate set partitions with the property that each block (when sorted) is parity alternating (i.e. odd, even, odd, etc.). We show that the total number of parity 
alternating partitions of the set $[m]$, satisfies the formula $a(m)=B_{\lfloor(m+1) / 2\rfloor} B_{\lceil(m+1) / 2\rceil}$, where $B_{k}$ is the $k$-th Bell number, the number of partitions of $[k]$. Note that the latter formula is not an obvious fact from the definition of $a(m)$. We apply the composition of operators in Weyl algebra to compute the number of such decompositions. This approach is illustrated for a new special type of set partitions, which we call the residue alternating partitions (a general version of the parity alternating partitions). The elements in every block of these partitions form a consecutive (cyclic) interval modulo $n$ and their total number is the product of Bell numbers. We show both algebraic and bijective proofs to this fact.

We then specify our interpretations to the case $n=1$, for which there are many related studies, e.g. $[3,4,12,18,20,21,24,30]$ and refer to a recent book [22] on the subject of normal ordering. We introduce and study the $\lambda$-Stirling numbers. These numbers of second kind naturally appear in decompositions of graphs with one vertex and many loops. For the given sequence $\lambda=\left(\lambda_{1} \geqslant \lambda_{2} \geqslant \cdots\right)$, the $\lambda$-Stirling numbers of the second kind $S_{\lambda}(n, k)$ is the number of partitions of $[n]$ into $k$ blocks such that the first $\lambda_{1}$ elements of $[n]$ are in distinct blocks, the next $\lambda_{2}$ elements are in distinct blocks, and so on. This definition is a natural generalization of the $r$-Stirling numbers [5] and it was studied in [25]. We show how these numbers $S_{\lambda}(n, k)$ arise from our general graph setting as well as from differential operators in the first Weyl algebra. On the other hand, we also define the corresponding dual $\lambda$-Stirling numbers of the first kind $C_{\lambda}(n, k)$, and show a combinatorial interpretation to them. Namely, $C_{\lambda}(n, k)$ is the number of permutations of $[n]$ having $k$ cycles such that non-minimal elements of the first $\lambda_{1}$ cycles are greater than all minimal elements of these $\lambda_{1}$ cycles; non-minimal elements of the next $\lambda_{2}$ cycles are greater than all minimal elements of these $\lambda_{2}$ cycles, and so on (some of the remaining cycles are singletons). The classical Stirling numbers are defined on two parameters: the number of elements and the number of blocks (cycles). For these generalizations, one can see that the $\lambda$-sequence affects on the first parameter in $\lambda$-Stirling numbers of the second kind, and affects on the second parameter (cycles) in $\lambda$-Stirling numbers of the first kind. We obtain many properties of $S_{\lambda}(n, k), C_{\lambda}(n, k)$ analogous to the properties of the usual Stirling numbers.

\section{Decompositions into increasing walks}

Suppose that edges of the digraph $G=(V, E)$ are ordered (or labeled), $E=\left(e_{1}, \ldots, e_{m}\right)$. A $k$-decomposition is a decompositions of $E$ into $k$ edge-disjoint walks. We say that the $k$-decomposition $E=P_{1} \cup \ldots \cup P_{k}$ is principal if every walk $P_{i}=e_{\ell_{1}} \ldots e_{\ell_{s}}(1 \leqslant i \leqslant k)$ is increasing, i.e. we have $\ell_{1}<\ldots<\ell_{s}$.

When $V=\{1\}$ and graph has $m$ labeled loop edges $(1,1)$, principal decompositions correspond to partitions of the set $[m]$ into disjoint subsets. Further, we suppose that the digraph $G$ is presented by the vertex set $V=[n]$.

A block (or $p$-block if $p$ is specified) is a distinguished set of edges $\left\{e_{1}, \ldots, e_{p}\right\}$. If graph is built up from several (disjoint) blocks, then we will require that the edges in each block must lie in distinct walks. 


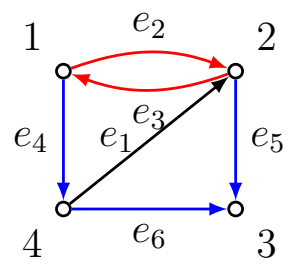

Figure 2: A digraph built of three edge blocks (red, blue, black).

For example, digraph in Figure 2 which is built from three blocks $B_{1}=\left\{e_{1}\right\}, B_{2}=$ $\left\{e_{2}, e_{3}\right\}, B_{3}=\left\{e_{4}, e_{5}, e_{6}\right\}$, has a principal 4-decomposition $e_{1} e_{5} \cup e_{2} \cup e_{3} e_{4} \cup e_{6}$. Note that decomposition $e_{1} e_{5} \cup e_{2} e_{3} e_{4} \cup e_{6}$ cannot be used here since $e_{2}, e_{3}$ are from the same block $B_{1}$ and thus cannot be in the same path.

For each vertex $v \in V$ consider the sets

$$
\operatorname{In}(v)=\left\{e_{i_{1}}, \ldots, e_{i_{s}}\right\}, \quad \operatorname{Out}(v)=\left\{e_{j_{1}}, \ldots, e_{j_{\ell}}\right\}
$$

of its incoming and outcoming edges, respectively (loops $(v, v)$ are included in both sets). Suppose that the edges of $G$ are built up from some partition of $E$ into blocks. Consider the matchings between $\operatorname{In}(v)$ and $\operatorname{Out}(v)$ defined as follows. We allow elements $e_{i} \in \operatorname{In}(v)$ and $e_{j} \in \operatorname{Out}(v)$ to be matched if $i<j$ and $e_{i}, e_{j}$ are not in the same block. A matching now is defined as some set of such matched pairs, where every edge is used at most once from $\operatorname{In}(v)$ and at most once from $\operatorname{Out}(v)$ (so, only loops can be used once for both $\operatorname{In}(v)$, $\operatorname{Out}(v)$ ). Let $\mathcal{M}(v)$ be the set of all possible matchings (not necessarily maximal, and including an empty matching) and $M(v)$ be the set of maximal matchings. For example, at vertex 2 of the graph in Figure 2 we have

$$
\begin{gathered}
\operatorname{In}(2)=\left\{e_{1}, e_{2}\right\}, \quad \operatorname{Out}(2)=\left\{e_{3}, e_{5}\right\}, \\
\mathcal{M}(2)=\left\{\emptyset,\left\{\left(e_{1}, e_{3}\right)\right\},\left\{\left(e_{1}, e_{5}\right)\right\},\left\{\left(e_{2}, e_{5}\right)\right\},\left\{\left(e_{1}, e_{3}\right),\left(e_{2}, e_{5}\right)\right\}\right\}, \\
M(2)=\left\{\left(e_{1}, e_{3}\right),\left(e_{2}, e_{5}\right)\right\} .
\end{gathered}
$$

Let $\mathcal{P} \mathcal{D}(G)$ be the set of principal decompositions of $G$.

Proposition 2.1. There is a bijection between the sets $\mathcal{M}(1) \times \cdots \times \mathcal{M}(n)$ and $\mathcal{P} \mathcal{D}(G)$.

Proof. Let us take an arbitrary matching for every vertex $v(1 \leqslant v \leqslant n)$ and construct a principal graph decomposition. If $e_{i} \in \operatorname{In}(v)$ and $e_{j} \in \operatorname{Out}(v)$ are matched, then we define them to be a fragment of a path $e_{i} e_{j}$. Otherwise, if $e_{i} \in \operatorname{In}(v)$ or $e_{j} \in \operatorname{Out}(v)$ are unmatched edges, then define them as final and initial edges of their corresponding paths, respectively. One can easily verify that this map defines a principal decomposition and its inverse defines a matching for every vertex.

Corollary 2.2. $|\mathcal{P} \mathcal{D}(G)|=\prod_{i=1}^{n}|\mathcal{M}(i)|$.

Denote $\operatorname{in}(v)=|\operatorname{In}(v)|, \operatorname{out}(v)=|\operatorname{Out}(v)|$. 
Corollary 2.3. If $G$ is acyclic digraph, then there exists a labeling of its edges such that every path is increasing. Therefore, every decomposition of $G$ is a principal decomposition for such labeling. For every vertex $v$ we can match some $i$ edges from $\operatorname{In}(v)$ with some $i$ edges of $\operatorname{In}(v)$ in $\left(\begin{array}{c}\operatorname{in}(v) \\ i\end{array}\right)\left(\begin{array}{c}\text { out }(v) \\ i\end{array}\right) i$ ! ways. Therefore,

$$
\mathcal{M}(v)=\sum_{i \geqslant 0}\left(\begin{array}{c}
\operatorname{in}(v) \\
i
\end{array}\right)\left(\begin{array}{c}
\operatorname{out}(v) \\
i
\end{array}\right) i !
$$

and the total number of decompositions of the acyclic digraph $G$ is

$$
\prod_{v=1}^{n}\left(\sum_{i \geqslant 0}\left(\begin{array}{c}
\operatorname{in}(v) \\
i
\end{array}\right)\left(\begin{array}{c}
\operatorname{out}(v) \\
i
\end{array}\right) i !\right) .
$$

\subsection{On general walk and path decompositions}

For a digraph $G=(V, E)$, define $\operatorname{flux}(v):=\operatorname{in}(v)-\operatorname{out}(v), v \in V$,

$$
V^{+}:=\{v \in V \mid \operatorname{flux}(v)>0\}, \quad V^{-}:=\{v \in V \mid \operatorname{flux}(v)<0\}
$$

and the total flux as

$$
f(G):=\sum_{v \in V^{+}} \operatorname{flux}(v)=\sum_{v \in V^{-}}-\operatorname{flux}(v) .
$$

The basic properties of walk decompositions such as existence criteria, simply rely on the usual Euler tours. We just add a new vertex so that for all $v \in V$ we get $\operatorname{flux}(v)=0$. This also allows to compute (or bound) the total number of walk decompositions using the BEST theorem and the Matrix-tree theorem.

Decompositions into walks have the following matroid structure studied in [23]. Sets of edges that are in distinct walks in a certain (minimal) decomposition are independent sets of a matroid of rank $f(G)$ (adding with the number of components of $G$ ). This matroid is isomorphic to a cotransversal matroid. In fact, the sets of source (initial) edges of all $f(G)$-decompositions also form a collection of matroid bases.

For (simple) path decompositions there are many studies (e.g. [1, 2, 8, 13, 16, 17, 19]), most of which are around Gallai's conjecture. Note that $f(G)$ is attainable minimal number of paths in a decomposition for acyclic digraphs; it was also shown in [1] that the minimal number of paths needed to decompose a transitive tournament digraph on $n$ vertices is $\left\lfloor n^{2} / 4\right\rfloor$. Gallai's conjecture states that every connected simple undirected graph with $n$ vertices can be decomposed into at most $\lceil n / 2\rceil$ paths; it is known that every such graph can be decomposed into at most $\lfloor n / 2\rfloor$ paths or cycles [19].

\section{$3 \quad$ G-Stirling functions}

We use the following notation for multisets: $A-X$ is a difference, e.g. $\left\{1^{3}, 2^{2}, 3,4^{3}\right\}-$ $\left\{1^{2}, 2,4\right\}=\left\{1,2,3,4^{2}\right\} ; A \uplus X$ is a merge, e.g. $\left\{1^{2}, 2,4^{2}\right\} \uplus\left\{1,2^{2}, 3\right\}=\left\{1^{3}, 2^{3}, 3,4^{2}\right\}$. We also write $G-e$ if edge $e$ is eliminated from $G$ or $G-B$ if block $B$ is removed. 
For a given digraph $G$, let

$$
\begin{gathered}
V_{\text {out }}:=\left\{1^{\text {out }(1)}, \ldots, n^{\text {out }(n)}\right\}, \quad V_{\text {in }}:=\left\{1^{\text {in }(1)}, \ldots, n^{\text {in }(n)}\right\}, \\
M_{\text {out }}(G):=\left\{I \mid I \subseteq V_{\text {out }}\right\},
\end{gathered}
$$

i.e., $M_{\text {out }}$ is the set of all $\operatorname{sub}\left(\right.$ multi)sets of $V_{\text {out }}$.

Note that if for a $k$-decomposition, we have the sources $I$, then the corresponding sinks $J=V_{\text {in }} \uplus I-V_{\text {out }}$ are determined uniquely. (Further, for any sources $I$ we will just write sinks as $J$ meaning that $J=V_{\text {in }} \uplus I-V_{\text {out. }}$.)

Define the $G$-Stirling function $S_{G}: M_{\text {out }}(G) \rightarrow \mathbb{Z}_{\geqslant 0}$ as follows

$$
S_{G}(I):=\text { the number of principal decompositions of } G \text { with sources } I \text {. }
$$

If $n=1$, then $S_{G}(I)$ corresponds to Stirling number of the second kind $S(m, k)$ where $|I|=k$ and digraph $G$ has $m$ labeled loops $(1,1)$.

Proposition 3.1. If $I=\left\{1^{\ell_{1}}, \ldots, n^{\ell_{n}}\right\}$, then

$$
S_{G}(I)=\prod_{i=1}^{n} S_{i}\left(\operatorname{out}(i)-\ell_{i}\right),
$$

where $S_{i}(k)$ the number of matchings in $\mathcal{M}(i)$ of size $k$.

Proof. Since $S_{i}(k)$ the number of matchings in $\mathcal{M}(i)$ of size $k$, the vertex $i$ is unmatched $\operatorname{out}(i)-k$ times. Thus, from the bijection of Proposition 2.1, the vertex $i$ is used exactly out $(i)-\ell_{i}$ times as a source. Therefore, we get the formula by considering this argument for every vertex $i=1, \ldots, n$ and using Proposition 2.1.

Theorem 3.2 ([15]). The G-Stirling function $S_{G}$ satisfies the following properties:

(i) $S_{G}\left(V_{\text {out }}\right)=1$;

(ii) if $S_{G}(I)>0$ for some $I \subset V_{\text {out }}$, then for any $I^{\prime}$, such that $V_{\text {out }} \supseteq I^{\prime} \supset I$, we have $S_{G}\left(I^{\prime}\right)>0$;

(iii) Suppose that digraph $G$ is built up from blocks $B_{1}, \ldots, B_{m}$ so that the indices of edges increase with respect to the order of blocks. Let $e=(i, j) \in B_{m}, G^{\prime}=G-e$, $I^{\prime}=I-\{i\}$. Let $k_{i}$ be the number of repetitions of $i$ in $(J-\{j\}) \uplus\{i\}$ and $r_{e}$ be the number of edges in $B_{m}-e$ that end by $i$. Then the following recurrence relation holds for $S_{G}(I)$.

$$
S_{G}(I)=S_{G^{\prime}}\left(I^{\prime}\right)+\left(k_{i}-r_{e}\right) S_{G^{\prime}}(I) .
$$

Corollary 3.3. If $G$ has edges $e_{1}, \ldots, e_{m}$ (without blocks), then for $e_{m}=(i, j), G^{\prime}=$ $G-e_{m}, I^{\prime}=I-\{i\}$ and $k_{i}$ the number of repetitions of $i$ in $(J-\{j\}) \uplus\{i\}$, we have

$$
S_{G}(I)=S_{G^{\prime}}\left(I^{\prime}\right)+k_{i} S_{G^{\prime}}(I) .
$$


Corollary 3.4. If $G$ has $n=1$ vertex and $m$ loops $(1,1)$, then $S_{G}(I)=S(m, k)$ if $|I|=k$, where $S(m, k)$ is Stirling number of the second kind. Relation (3) becomes the well-known recurrence

$$
S(m, k)=S(m-1, k-1)+k S(m-1, k) .
$$

Remark 3.5. The G-Stirling function $S_{G}(I)$ is a graph generalization of Stirling number of the second kind. Note that $S_{G}$ is different from Stirling (and Bell) numbers for graphs studied in [14], which count partitions of graph vertex set into independent sets. Although, for $n=1$ (and several blocks) there is a correspondence between these definitions as noted in section 6 .

In fact, $M_{\text {out }}(G)$ can be considered as a poset ordered by inclusion. Let us consider a subdomain of $M_{\text {out }}(G)$ at which $S_{G}$ takes positive values; define the poset

$$
P_{G}:=\left\{I \mid S_{G}(I)>0\right\}
$$

whose elements (multisets) are ordered by inclusion.

Proposition 3.6. Let $m(v)=|M(v)|$ be the size of a maximal matching. Then

(i) $P_{G}$ has a unique maximal element $V_{\text {out }}$, unique minimal element $V_{0}$, where

$$
V_{0}=V_{\text {out }}-\left\{1^{m(1)}, \ldots, n^{m(n)}\right\} .
$$

(ii) $P_{G}$ is isomorphic to $\mathbf{m}(\mathbf{1}) \times \cdots \times \mathbf{m}(\mathbf{n})$.

Here by $\mathbf{m}$ we denote the chain poset of $m$ elements and $\mathbf{m} \times \mathbf{s}$ is the poset of $m s$ elements defined as (cartesian) product of posets $\mathbf{m}, \mathbf{s}$ (if $\mathbf{s}$ is empty, then put $\mathbf{m} \times \mathbf{s}=\mathbf{m}$ ). (See e.g. [29])

Proof. First, from (i), (ii) of Theorem 3.2, $V_{\text {out }}$ is a unique maximal element and if $V_{0}$ is some minimal element, then for all $V_{0} \subseteq I \subseteq V_{\text {out }}$. Let us prove that

$$
V_{0}=\left\{1^{\text {out }(1)-m(1)}, \ldots, n^{\text {out }(n)-m(n)}\right\}
$$

is a unique minimal element. From Theorem 2.1, there is a principal decomposition with sources $V_{0}$, so $V_{0} \in P_{G}$. If there is another minimal element $V^{\prime}$, then $i^{\text {out }(i)-m(i)} \notin V^{\prime}$ for some $i$. This means that the vertex $i$ has a matching of size greater than $m(i)$, which is impossible. So, both items (i), (ii) clearly imply from these arguments.

Corollary 3.7. If $G$ is a cycle graph with edge labels given by

$$
1 \rightarrow^{e_{1}} 2 \rightarrow^{e_{2}} \cdots \rightarrow^{e_{n-1}} n \rightarrow^{e_{n}} 1,
$$

then $m(1)=0$, and $m(i)=2$ for $i=2, \ldots, n$. Hence, $P_{G}$ is isomorphic to $\mathbf{2} \times \cdots \times \mathbf{2}=$ $2^{[n-1]}$. 
The total number of principal decompositions $|\mathcal{P D}(G)|$ serves as a generalized Bell number. Let $B_{G}=|\mathcal{P} \mathcal{D}(G)|$, then we have

$$
B_{G}=\sum_{V_{0} \subseteq I \subseteq V_{\text {out }}} S_{G}(I) .
$$

If we now define an extension $B_{G}(J)$ as

$$
B_{G}(J):=\sum_{V_{0} \subseteq I \subseteq J} S_{G}(I)
$$

then applying the Möbius inversion formula on the poset $P_{G}$ we get

$$
S_{G}(J)=\sum_{I \subseteq J} \mu(I, J) B_{G}(I)
$$

and in particular

$$
S_{G}\left(V_{\text {out }}\right)=1=\sum_{I \subseteq V_{\text {out }}} \mu(I, J) B_{G}(I) .
$$

Note that $P_{G}$ is isomorphic to a divisibility poset, and $\mu(I, J)=(-1)^{|J-I|}$ if $J-I$ is a set and 0 if $J-I$ is a multiset. Hence we get

$$
S_{G}(J)=\sum_{I \subseteq[n]}(-1)^{|J-I|} B_{G}(J-I)
$$

and the kind of recurrence

$$
B_{G}=1-\sum_{\emptyset \subset I \subseteq[n]}(-1)^{\left|V_{\text {out }}-I\right|} B_{G}\left(V_{\text {out }}-I\right) .
$$

\section{Normal ordering in the Weyl algebra}

Let $K$ be a field of characteristic 0 . The $n$-th Weyl algebra $A_{n}$ is an associative algebra over $K$ defined by $2 n$ generators $x_{1}, \ldots, x_{n}, \partial_{1}, \ldots, \partial_{n}$ and relations

$$
\left[x_{i}, x_{j}\right]=\left[\partial_{i}, \partial_{j}\right]=0,\left[\partial_{i}, x_{j}\right]=\delta_{i, j} \text { for } 1 \leqslant i, j \leqslant n,
$$

where $[a, b]=a b-b a$ is the commutator and $\delta_{i, j}$ is the Kronecker symbol. The typical example of $A_{n}$ is the polynomial algebra with $\partial_{i}$ considered as partial derivations $d / d x_{i}$. The elements of types

$$
x^{\alpha} \partial^{\beta}:=x_{1}^{\alpha_{1}} \cdots x_{n}^{\alpha_{n}} \partial_{1}^{\beta_{1}} \cdots \partial_{n}^{\beta_{n}}
$$

with $\alpha=\left(\alpha_{1}, \ldots, \alpha_{n}\right), \beta=\left(\beta_{1}, \ldots, \beta_{n}\right) \in \mathbb{Z}_{\geqslant 0}^{n}$ are called monomials. We will also write monomials in the equivalent form $x_{i_{1}} \ldots x_{i_{s}} \partial_{j_{1}} \ldots \partial_{j_{p}}$. All monomials $x^{\alpha} \partial^{\beta}$ form a linear vector space basis of $A_{n}$. When the element $w$ of $A_{n}$ is expressed as a linear combination

$$
w=\sum_{\alpha, \beta} c(\alpha, \beta) x^{\alpha} \partial^{\beta}, \quad c(\alpha, \beta) \in K,
$$


we say that $w$ is normally ordered. Define the following subspaces of $A_{n}$ :

$$
A_{n}^{(p, q)}:=\left\langle x^{\alpha} \partial^{\beta}:|\alpha|=p,|\beta|=q\right\rangle, \quad A_{n}^{(0)}:=\bigoplus_{i \geqslant 1} A_{n}^{(i, i)}
$$

where $|\alpha|=\sum_{i=1}^{n} \alpha_{i}$. Note that $A_{n}^{(0)}$ is the subalgebra of $A_{n}$.

We show that combinatorial meaning of coefficients in the normal ordering can be interpreted in terms of graph decompositions. Furthermore, we will consider the monomials of subspace $A_{n}^{(0)}$ (otherwise, we may add fictive elements as shown in subsection 6.1). We associate every monomial $w=x_{i_{1}} \ldots x_{i_{p}} \partial_{j_{1}} \ldots \partial_{j_{p}} \in A_{n}^{(0)}$ with the $p$-block of a graph in the following way:

$$
\operatorname{block}(w):=\left\{\left(i_{1}, j_{1}\right), \ldots,\left(i_{p}, j_{p}\right)\right\}
$$

Theorem 4.1 ([15]). Let $w_{1}, \ldots, w_{m} \in A_{n}^{(0)}$ be monomials. Then we have

$$
w_{1} \cdots w_{m}=\sum_{I \subseteq V_{\text {out }}} S_{G}(I) \prod_{i \in I} x_{i} \prod_{j \in J} \partial_{j}
$$

where $G=([n], E)$ with $E=\left(\operatorname{block}\left(w_{1}\right), \ldots, \operatorname{block}\left(w_{m}\right)\right)$ (i.e. the indices of edges increase with respect to the order of blocks) and $J=V_{\text {in }} \uplus I-V_{\text {out }}$.

Corollary 4.2. For a digraph $G=([n], E)$ with $E=\left(e_{1}, \ldots, e_{m}\right)$, we have

$$
\prod_{\ell=1}^{\vec{m}} x_{i_{\ell}} \partial_{j_{\ell}}=\sum_{I} S_{G}(I) \prod_{i \in I} x_{i} \prod_{j \in J} \partial_{j}
$$

where $e_{\ell}=\left(i_{\ell}, j_{\ell}\right)$ and the sum runs over all (multi)sets of sources $I$.

Corollary 4.3. If $n=1$, then (8) gives the well-known formula

$$
(x \partial)^{m}=\sum_{i=0}^{m} S(m, i) x^{i} \partial^{i} .
$$

where $S(m, i)$ is Stirling number of the second kind.

Analogous to formula (8), the operators $x_{i} \partial_{j}+x_{j} \partial_{i}$ give a decomposition formula for undirected graphs. Namely, we obtain the following result.

Theorem 4.4. For an undirected graph $G=([n], E)$ with $E=\left(e_{1}, \ldots, e_{m}\right), e_{\ell}=\left(i_{\ell}, j_{\ell}\right)$, we have

$$
\prod_{\ell=1}^{\vec{m}}\left(x_{i_{\ell}} \partial_{j_{\ell}}+x_{j_{\ell}} \partial_{i_{\ell}}\right)=\sum_{\begin{array}{c}
\text { decompositions into increasing } \\
\text { walks with sources I }
\end{array}} \prod_{i \in I} x_{i} \prod_{j \in J} \partial_{j} .
$$


The normal ordering decomposition formulas like (8), (9) are in particular useful when we sum through all decompositions of $G$ or symmetrize over all permutations of the edge set. For $w_{1}, \ldots, w_{m} \in A_{n}$, let

$$
s_{m}^{+}\left(w_{1}, \ldots, w_{m}\right):=\sum_{\sigma \in S_{m}} w_{\sigma(1)} \cdots w_{\sigma(m)} .
$$

Then for undirected graph $G$ we have

$s_{m}^{+}\left(x_{i_{1}} \partial_{j_{1}}+x_{j_{1}} \partial_{i_{1}}, \ldots, x_{i_{m}} \partial_{j_{m}}+x_{j_{m}} \partial_{i_{m}}\right)=($ \# Euler tours $i \rightarrow j) x_{i} \partial_{j}+$ higher order terms.

Consider a case of computing the sum $s_{m}^{+}$.

\section{Proposition 4.5.}

$$
\begin{aligned}
& s_{m}^{+}\left(x_{1} \partial_{2}, x_{2} \partial_{3}, \ldots, x_{m-1} \partial_{m}, x_{m} \partial_{m+1}\right) \\
& =x_{1}\left(\sum_{2 \leqslant i_{1}<\cdots<i_{k} \leqslant m}\left(\begin{array}{c}
m \\
i_{1}-1, i_{2}-i_{1}, \ldots, m+1-i_{k}
\end{array}\right) \prod_{\ell=1}^{k} x_{i_{\ell}} \prod_{\ell=1}^{k} \partial_{i_{\ell}}\right) \partial_{m+1},
\end{aligned}
$$

where $\left(\begin{array}{c}m \\ m_{1}, \ldots, m_{k}\end{array}\right)=\frac{m !}{m_{1} ! \cdots m_{k} !}$ is a multinomial coefficient.

Proof. From monomials $x_{1} \partial_{2}, x_{2} \partial_{3}, \ldots, x_{m-1} \partial_{m}, x_{m} \partial_{m+1}$, the digraph $G$ is a single path $1 \rightarrow 2 \rightarrow \cdots \rightarrow m \rightarrow m+1$. Taking all permutations $\sigma \in S_{m}$ means that we permute the edges of $G$ up to $\sigma$. Consider decompositions into increasing walks in that case. Suppose that we break the paths at vertices $2 \leqslant i_{1}<\cdots<i_{k} \leqslant m$. Then the number of permutations $\sigma \in S_{m}$ for which the fragments $1 \rightarrow \cdots \rightarrow i_{1} / i_{1} \rightarrow \cdots \rightarrow i_{2} / \cdots / i_{k} \rightarrow$ $\cdots \rightarrow m+1$ are all increasing, is clearly equal to $\left(\begin{array}{c}m \\ i_{1}-1, i_{2}-i_{1}, \ldots, m+1-i_{k}\end{array}\right)$ and the corresponding monomial is $x_{1} x_{i_{1}} \cdots x_{i_{k}} \partial_{i_{1}} \cdots \partial_{i_{k}} \partial_{m+1}$ and so we obtain the formula.

Remark 4.6. Note that the last formula is computationally effective; we compute $2^{m-1}$ terms instead of calculating $s_{m}^{+}$with $m$ ! summands.

\section{Cyclic multigraphs and residue alternating partitions}

Consider the digraph $C_{m, n}$ with $n$ vertices and $m$ edges $(m>n)$ that consecutively form a cycle $(12 \cdots n)$, i.e. the edges $\left\{e_{1}, \ldots, e_{m}\right\}$ in order are

$$
e_{1}=(1,2), e_{2}=(2,3), \ldots, e_{n}=(n, 1), e_{n+1}=(1,2), \ldots
$$

The principal decompositions on such graph correspond to the following type of set partitions. Say that the set partition $\cup X_{i}=[m]$ is residue alternating if for every $i(1 \leqslant i \leqslant k)$ and $X_{i}=\left\{a_{1}, \ldots, a_{r}\right\}$ with $a_{1}<\cdots<a_{r}$, we have $a_{i+1}-a_{i} \equiv 1(\bmod n)$ for $i=1, \ldots, r$ $\left(a_{r+1}=a_{1}\right)$.

Let $A(m, n)$ be the number of residue alternating partitions (or the number of decompositions of digraph $\left.C_{m, n}\right)$. 
Theorem 5.1. The following formulas hold for $A(m, n)$

$$
A(n k, n)=B_{k}\left(B_{k+1}\right)^{n-1}
$$

and for $0<r<n$

$$
A(n k+r, n)=\left(B_{k+1}\right)^{n-r+1}\left(B_{k+2}\right)^{r-1},
$$

where $B_{k}$ is the Bell number, the number of partitions of set $[k]$.

Proof. First we examine the approach using composition of differential operators. Note that $A(m, n)$ corresponds to the number $B_{G}=\sum_{I} S_{G}(I)$ (see def. (5)) of all principal decompositions of $G=C_{m, n}$. Therefore, we can use the normal ordering expansion of differential operators to calculate $A(m, n)=B_{G}$.

If $m=n k$, then the composition of operators is

$$
\left(x_{1} \partial_{2} \cdots x_{n} \partial_{1}\right) \cdots\left(x_{1} \partial_{2} \cdots x_{n} \partial_{1}\right)=\left(x_{1} \partial_{2} \cdots x_{n} \partial_{1}\right)^{k}=\left(x_{1} \partial_{1}\right)^{k}\left(\partial_{2} x_{2}\right)^{k} \cdots\left(\partial_{n} x_{n}\right)^{k} .
$$

In fact, $A(m, n)$ is the sum of coefficients in the normal ordering expansion of the last expression. The sum of coefficients in expansion $\left(x_{1} \partial_{1}\right)^{k}$ is

$$
\sum_{i=0}^{k} S(k, i)=B_{k}
$$

and the sum of coefficients in the normal ordering of $\left(\partial_{\ell} x_{\ell}\right)^{k}$ is

$$
\sum_{i=0}^{k}\left(\begin{array}{l}
k \\
i
\end{array}\right) B_{i}
$$

since

$$
\left(\partial_{\ell} x_{\ell}\right)^{k}=\left(1+x_{\ell} \partial_{\ell}\right)^{k}=\sum_{i=0}^{k}\left(\begin{array}{l}
k \\
i
\end{array}\right)\left(x_{\ell} \partial_{\ell}\right)^{i} .
$$

It remains to use the well-known recurrence for Bell numbers

$$
B_{k+1}=\sum_{i=0}^{k}\left(\begin{array}{l}
k \\
i
\end{array}\right) B_{i} .
$$

So, the sum of coefficients $A(n k, n)$ in the normal ordering of $\left(x_{1} \partial_{1}\right)^{k}\left(\partial_{2} x_{2}\right)^{k} \cdots\left(\partial_{n} x_{n}\right)^{k}$ is $B_{k}\left(B_{k+1}\right)^{n-1}$.

Similarly, if $m=n k+r$ we have the composition

$$
\begin{aligned}
& \left(x_{1} \partial_{2} \cdots x_{n} \partial_{1}\right) \cdots\left(x_{1} \partial_{2} \cdots x_{n} \partial_{1}\right) x_{1} \partial_{2} \cdots x_{r} \partial_{r+1} \\
& =x_{1}\left(\partial_{1} x_{1}\right)^{k}\left[\left(\partial_{2} x_{2}\right)^{k+1} \cdots\left(\partial_{r} x_{r}\right)^{k+1}\right]\left(\partial_{r+1} x_{r+1}\right)^{k} \partial_{r+1}\left[\left(\partial_{r+2} x_{r+2}\right)^{k} \cdots\left(\partial_{n} x_{n}\right)^{k}\right]
\end{aligned}
$$

and by the same argument it follows that $A(n k+r, n)=\left(B_{k+1}\right)^{n-r+1}\left(B_{k+2}\right)^{r-1}$. 
Bijective proof. Using Corollary 2.2 of Theorem 2.1 we look at matchings of edges at every vertex.

Suppose $m=n k$ and consider the vertex $i$, where $2 \leqslant i \leqslant n$. We have

$$
\begin{gathered}
\operatorname{In}(i)=\left\{e_{i-1}, e_{n+i-1}, e_{2 n+i-1}, \ldots, e_{(k-1) n+i-1}\right\}, \\
\operatorname{Out}(i)=\left\{e_{i}, e_{n+i}, e_{2 n+i}, \ldots, e_{(k-1) n+i}\right\} .
\end{gathered}
$$

We will prove that $|\mathcal{M}(i)|=B_{k+1}$ by establishing a bijection between matchings in $\mathcal{M}(i)$ and partitions of set $[k+1]$. Let $M \in \mathcal{M}(i)$ be any matching between $\operatorname{In}(i) \operatorname{Out}(i)$. Construct set partition of $[k+1]$ as follows:

(1) if $\left(e_{a n+i-1}, e_{b n+i}\right) \in M$, where $a \leqslant b$, then put $a+1, b+2$ in the same block;

(2) the remaining elements of $[k+1]$ (that were not considered yet), put in separate blocks.

For example, if $k=5, \operatorname{In}(i)=\left\{e_{1}, e_{3}, e_{5}, e_{7}, e_{9}\right\}$, Out $(i)=\left\{e_{2}, e_{4}, e_{6}, e_{8}, e_{10}\right\}$,

$$
M=\left\{\left(e_{1}, e_{6}\right),\left(e_{3}, e_{4}\right),\left(e_{5}, e_{8}\right)\right\},
$$

then $\left(e_{1}, e_{6}\right)$ means that we should put 1,4 in the same block; for $\left(e_{3}, e_{4}\right)$ the elements 2,3 are in the same block; and for $\left(e_{5}, e_{8}\right)$ the elements 3,5 are in the same block. Therefore, we have the partition $\{1,4\}\{2,3,5\}\{6\}$.

The inverse procedure can be described as follows: take any block of the partition, $\left\{a_{1}<\cdots<a_{r}\right\}$ and for every $j=1, \ldots, r-1$ match the edges $\left(e_{\left(a_{j}-1\right) n+i-1}, e_{\left(a_{j+1}-2\right) n+i}\right)$.

One can see that this properly defines the bijection.

By applying a similar argument one can show that $|\mathcal{M}(1)|=B_{k}$ and therefore, by Theorem 2.1, $A(n k, n)=B_{k}\left(B_{k+1}\right)^{n-1}$. The formula for $A(n k+r, n)$ implies analogously.

\subsection{Parity alternating partitions}

For $n=2$ we have the graph model with 2 vertices and $n$ edges $\left\{e_{1}, \ldots, e_{n}\right\}$ such that all odd-indexed $e_{2 i-1}$ are of type $(1,2)$ and all even-indexed $e_{2 i}$ are of type $(2,1)$.

All principal decompositions on this graph can be considered as partitions $X_{1} \cup \cdots \cup X_{k}$ of $[m]$ that have the following property: For every $i(1 \leqslant i \leqslant k)$ and $X_{i}=\left\{a_{1}, \ldots, a_{r}\right\}$ with $a_{1}<\cdots<a_{r}$, the sequence $a_{1}, \ldots, a_{r}$ is parity alternating (i.e. even, odd, even, odd, etc. or similarly beginning with odd). Let us call such partitions of sets parity alternating partitions. For example, the parity alternating partitions of $\{1,2,3,4\}$ into two blocks are

$$
\{1\}\{2,3,4\} ; \quad\{4\}\{1,2,3\} ; \quad\{1,2\}\{3,4\} ; \quad\{1,4\}\{2,3\} .
$$

Denote by $a(m)=A(m, 2)$ the total number of parity alternating partitions of $[m]$. Then the following formulas hold

$$
a(2 k)=B_{k} B_{k+1}, \quad a(2 k+1)=B_{k+1}^{2} .
$$


Remark 5.2. The latter formulas mean that the number of parity alternating partitions of $[m]$ is equal to the number of partitions of $[m+1]$ where elements in each block have the same parity. (The second sequence appears in OEIS, A124419 [28]). Bijectively, this fact can be described as follows: $x, y$ are successive elements in the block of parity alternating partitions iff $x, y+1$ are successive elements of the second type of partitions. Note that such reduction algorithm was applied to regular (noncrossing) set partitions in [6].

The values of $a(m)$ can also be computed by the number of blocks. Let $a(m, k)$ be the number of parity alternating partitions into $k$ blocks and $a(m, k, i)$ the number of parity alternating partitions into $k$ blocks $i$ of which have even maximal elements. We have $a(m, k)=\sum_{i=0}^{k} a(m, k, i), a(m)=\sum_{k=1}^{n} a(m, k)$.

\begin{tabular}{c|llllllll|c}
$m \backslash k$ & 1 & 2 & 3 & 4 & 5 & 6 & 7 & 8 & $a(m)$ \\
\hline 1 & 1 & & & & & & & & 1 \\
2 & 1 & 1 & & & & & & 2 \\
3 & 1 & 2 & 1 & & & & & & 4 \\
4 & 1 & 4 & 4 & 1 & & & & & 10 \\
5 & 1 & 6 & 11 & 6 & 1 & & & 25 \\
6 & 1 & 10 & 28 & 26 & 9 & 1 & & & 75 \\
7 & 1 & 14 & 61 & 86 & 50 & 12 & 1 & & 225 \\
8 & 1 & 22 & 136 & 276 & 236 & 92 & 16 & 1 & 780
\end{tabular}

Table 1: Small values of $a(m)$ and $a(m, k)$.

Proposition 5.3. Recurrence relations for $a(m, k, i)$ are given by

$$
\begin{gathered}
a(1,1,0)=1, a(m, k, i)=0 \text { for } m<k \text { or } k<i, \\
a(2 m+1, k, i)=a(2 m, k-1, i)+(i+1) a(2 m, k, i+1), \\
a(2 m, k, i)=a(2 m-1, k-1, i-1)+(k-i+1) a(2 m-1, k, i-1) .
\end{gathered}
$$

Proof. Consider parity alternating partitions of set $[2 m+1]$ into $k$ blocks, $i$ of whose maximal elements are even. The element $2 m+1$ can form a separate block contributing $a(2 m, k-1, i)$ ways. Otherwise, $2 m+1$ can be placed into blocks of parity alternating partitions of $[2 m]$ having $k$ blocks and $(i+1)$ maximal even elements (since $2 m+1$ will change the parity of one maximal element); this gives $(i+1) a(2 m, k, i+1)$ ways.

Similarly, if we look at partitions of $[2 m]$, then $2 m$ can form a separate block in $a(2 m-1, k-1, i-1)$ ways and can be placed into other blocks in $(k-i+1) a(2 m-1, k, i-1)$ ways.

\section{Case $n=1$, the $\lambda$-Stirling numbers}

\subsection{Normal ordering for $n=1$.}

Combinatorial interpretations of coefficients in the normal ordering expansion

$$
x^{r_{1}} \partial^{s_{1}} \cdots x^{r_{t}} \partial^{s_{t}}=\sum_{i} S_{G}(i) x^{i} \partial^{j}
$$


can be extracted from Theorem 4.1 as follows. The elements $x^{r_{i}} \partial^{s_{i}}$ are not from $A_{1}^{(0)}=$ $\left\langle x^{i} \partial^{i}: i=0,1, \ldots\right\rangle$ in general (i.e. if $r_{i} \neq s_{i}$ ). To deal with this situation, we add fictive $\left|r_{i}-s_{i}\right|$ new variables $x_{i+1}$ or $\partial_{i+1}$ so that the monomial will belong to $A_{1}^{(0)}$, and the graph scheme can be applied. For example, $x^{2} \partial^{5}$ is transformed to $x_{2}^{3} x^{2} \partial^{5}$. Note that the new variables commute with all other and so we can freely move them in the normal ordering expansion. Using these new monomials, we construct the graph $G$ according to the rules above. Thus, combinatorial meaning of $S_{G}(i)$ can be described as the number of principal decompositions of $G$ having $i$ sources at vertex 1 . This interpretation is similar to the graph models studied in [3] (the model there is acyclic which is different to ours since for $n=1$ we have loops).

Remark 6.1. In fact, the normal ordering in the $n$-th Weyl algebra can be computed using the $n=1$ case. For instance, we can restructure compositions as follows

$$
x_{1} x_{2} \partial_{1} \partial_{3} x_{2} x_{3} \partial_{1} \partial_{3} x_{1} \partial_{2}=\left(x_{1} \partial_{1}^{2} x_{1}\right)\left(x_{2}^{2} \partial_{2}\right)\left(\partial_{3} x_{3} \partial_{3}\right) .
$$

This view also helps to refine all possible multisets of sources and sinks $I, J$, since what coefficients are nonzero in every composition like (10) can be found.

\subsection{The $\lambda$-Stirling numbers}

Consider the graph with $n=1$ vertex and suppose it is built up from blocks of loops $(1,1)$ of $\lambda_{1}, \lambda_{2}, \ldots$ edge sizes. Principal decompositions on this model require that the edges within one block cannot lie on the same walk. This setting clearly corresponds to partitions of the set $[m]$ ( $m$ is a total number of edges), where the first $\lambda_{1}$ elements are in distinct subsets, the next $\lambda_{2}$ elements are also in distinct subsets, and so on. The coefficients $S_{G}(I)$ present a generalization of Stirling numbers of the second kind on such restricted partitions. In this section we study these generalized Stirling numbers. We also introduce a generalization of Stirling numbers of the first kind, which can be considered as dual to the second. We call these numbers the $\lambda$-Stirling numbers.

Fix the sequence

$$
\lambda_{0}=0, \quad \lambda=\left(\lambda_{1}, \lambda_{2}, \ldots\right), \quad \lambda_{1} \geqslant \lambda_{2} \geqslant \cdots
$$

of nonnegative integers, and let

$$
\begin{gathered}
q=q_{n}:=\max \left\{i \mid \lambda_{0}+\cdots+\lambda_{i} \leqslant n\right\}, \\
r=r_{n}:=n-\lambda_{0}-\cdots-\lambda_{q} .
\end{gathered}
$$

So $\lambda$ is a kind of 'infinite' integer partition, $q$ is the analog of quotient and $r$ is the analog of remainder.

Definition. The $\lambda$-Stirling numbers of second and first kinds $S_{\lambda}(n, k), C_{\lambda}(n, k)$ are defined as follows: 
- $S_{\lambda}(n, k)$ is the number of partitions of $[n]$ into $k$ blocks such that the first $\lambda_{1}$ elements of $[n]$ are in distinct blocks, the next $\lambda_{2}$ elements are in distinct blocks, and so on; the remaining $r_{n}$ elements are also in distinct blocks.

- $C_{\lambda}(n, k)$ is the number of permutations of $[n]$ with $k$ cycles such that non-minimal elements of the first $\lambda_{1}$ cycles are greater than all minimal elements of these $\lambda_{1}$ cycles; non-minimal elements of the next $\lambda_{2}$ cycles are greater than all minimal elements of these $\lambda_{2}$ cycles, and so on; and the remaining $r_{k}$ cycles are singletons (i.e. consist of one element).

By definition, the sequence $\lambda$ affects on the first argument $n$ in $S_{\lambda}(n, k)$ and on the second argument $k$ in $C_{\lambda}(n, k)$. Further we will see that $S_{\lambda}(n, k), C_{\lambda}(n, k)$ are dual to each other.

Remark 6.2. For permutation $\sigma$ of $[n]$ having $k$ cycles we associate representation in the form $\sigma=\left(\sigma^{(1)}\right) \cdots\left(\sigma^{(k)}\right)$, where $\sigma^{(1)}, \ldots, \sigma^{(k)}$ are cycles written in increasing order of their minimal elements. For example, the permutation $(5,6,1,7,3,2,4)$ is written as $(1,5,3)(2,6)(4,7)$.

So we divide the set $[n]$ according to the integer partition $n=\lambda_{1}+\ldots+\lambda_{q}+r$ :

$$
[n]=\{\underbrace{1, \ldots, \lambda_{1}}_{1 \text { st part }}, \ldots, \underbrace{\lambda_{1}+\cdots+\lambda_{q-1}+1, \ldots, \lambda_{1}+\cdots+\lambda_{q}}_{q \text {-th part }}, \underbrace{n-\lambda_{q}+1, \ldots, n}_{\text {last } r \text { elements }}\}
$$

and consider only those set partitions which restrict the elements of the same part to be in the same subset.

For the case of permutations, we take the partition $k=\lambda_{1}+\ldots+\lambda_{q_{k}}+r_{k}$ and consider permutations of the following type:

$$
\underbrace{\sigma^{(1)}, \ldots, \sigma^{\left(\lambda_{1}\right)}}_{\forall \text { non-min }>\forall \text { min }}|\cdots| \underbrace{\sigma^{\left(\lambda_{1}+\cdots+\lambda_{q_{k-1}}+1\right)}, \ldots, \sigma^{\left(\lambda_{1}+\cdots+\lambda_{q_{k}}\right)}}_{\forall \text { non-min }>\forall \text { min }} \mid \underbrace{\sigma^{\left(k-r_{k}+1\right)}, \ldots, \sigma^{(k)}}_{\text {last } r_{k} \text { singletons }}
$$

Consider examples. If $\lambda=(3,2,1,1, \ldots)$, then $S_{\lambda}(6,4)=30$ and allowed configurations of partitions of $\{1,2,3,4,5,6\}$ into 4 blocks can be described as follows:

- If 1,2,3,4 are blocks minima; then 5 can be put with 1,2,3 (except restriction with 4) and 6 with $1,2,3,4$ which totally gives $3 \times 4=12$ ways;

- If $1,2,3,5$ are blocks minima; then 4 can be put with $1,2,3$ and 6 with $1,2,3,4$ which totally gives $3 \times 4=12$ ways;

- If 1,2,3,6 are blocks minima; then 4 can be put with 1,2,3 and 5 with 1,2,3 except the block with 4 , which totally gives $3 \times 2=6$ ways;

So, there are totally $12+12+6=30$ ways to arrange desired partitions.

$C_{\lambda}(6,4)=36$ and configurations of permutations of $(1, \ldots, 6)$ with 4 cycles can be described as follows:

(1,2,3 should always be cycle minima; and cycle number 4 is singleton) 
- If $1,2,3,4$ are cycle minima; then 5 can be put with $1,2,3$ and 6 can be put after $1,2,3,5$, which gives $3 \times 4=12$ ways;

- If $1,2,3,5$ are cycle minima; then 4 can be put with $1,2,3$ and 6 can be put after $1,2,3,4$, which gives $3 \times 4=12$ ways;

- If $1,2,3,6$ are cycle minima; then 5 can be put with $1,2,3$ and 4 can be put after $1,2,3,5$, which gives $3 \times 4=12$ ways.

So, there are totally $12+12+12=36$ possible permutations with 4 cycles.

Clearly if $\lambda=(1,1, \ldots)$, then $C_{\lambda}(n, k), S_{\lambda}(n, k)$ are just the usual Stirling numbers. The case $\lambda=(r, 1,1, \ldots)$ corresponds to the $r$-Stirling numbers of first and second kinds introduced in [5]. Generalized Stirling numbers of the second kind $S_{\mathbf{r}, \mathbf{s}}(n, k)$ that arise from the expansion

$$
x^{r_{1}} \partial^{s_{1}} \cdots x^{r_{n}} \partial^{s_{n}}=x^{\sum r_{i}-d_{i}} \sum_{k} S_{\mathbf{r}, \mathbf{s}}(n, k) x^{k} \partial^{k} .
$$

have been studied in the bosons normal ordering problem [4, 24] (see also [12]). Our definition gives a natural (and simple) combinatorial interpretation to the case $S_{\mathbf{r}, \mathbf{r}}(n, k)$ and also solves an inverse problem, where Stirling numbers of the first kind arise. Note that the general formulas for $S_{\mathbf{r}, \mathbf{s}}(n, k)$ given in [24] can be used to compute our numbers $S_{G}, B_{G}$ (defined in Section 2). Interpretation to $S_{r, r}(n, k)$ with colorings of complete graphs introduced in [7] is very close to the meaning of $S_{\lambda}(n, k)(\lambda=(r, r, \ldots))$, since any $r$ elements that cannot be in the same subset can be viewed as proper colorings of a component complete graph $K_{r}$; on the other hand, this definition corresponds to Stirling numbers for graphs in the sense of counting partitions of a vertex set into independent sets (see [14]). The numbers $S_{\lambda}(n, k)$ were introduced in [25] as $\left(r_{1}, \ldots, r_{p}\right)$-Stirling numbers of the second kind. In order to be consistent with the corresponding Stirling numbers of the first kind $C_{\lambda}(n, k)$, we define these numbers over a general sequence $\lambda$ relating it with integer partitions, since it somehow acts (as integer partition) on first (or resp. second) argument of these Stirling numbers. In fact, by polynomial relations (18), (19) shown below, the $\lambda$-Stirling numbers correspond to a case of the multiparameter noncentral Stirling numbers introduced in [11]. This also leads to a case of a general study of connection constants between persistent sequences of polynomials [10].

The numbers $S_{\lambda}(n, k)$ also related to compositions (ordered partitions) of multisets in the following way. For $n=\lambda_{1}+\cdots+\lambda_{q}+r$ consider a multiset

$$
\mathbf{n}:=\left\{1^{\lambda_{1}}, \ldots, q^{\lambda_{q}},(q+1)^{r}\right\} .
$$

Then the number of ways to distribute the elements of $\mathbf{n}$ into $k$ ordered nonempty sets is equal to

$$
\frac{S_{\lambda}(n, k) k !}{\lambda_{1} ! \cdots \lambda_{q} ! r !}
$$


This easily implies from the combinatorial interpretation of $S_{\lambda}(n, k)$. If blocks are ordered, then we multiply the number of ways by $k$ !. The elements $\lambda_{1}+\cdots+\lambda_{j-1}+1, \ldots, \lambda_{1}+\cdots+\lambda_{j}$ (which all in distinct blocks) can be changed to the repetition $j^{\lambda_{j}}$; that was calculated $\lambda_{j}$ ! times.

$$
\lambda=(2,2,1,1, \ldots)
$$

\begin{tabular}{c|ccccc}
$n \backslash k$ & 2 & 3 & 4 & 5 & 6 \\
\hline 2 & 1 & & & & \\
3 & 2 & 1 & & & \\
4 & 2 & 4 & 1 & & \\
5 & 4 & 14 & 8 & 1 & \\
6 & 8 & 46 & 46 & 13 & 1 \\
\multicolumn{5}{c}{$S_{\lambda}(n, k)$ for $2 \leqslant n, k \leqslant 6$}
\end{tabular}

\begin{tabular}{c|ccccc}
$n \backslash k$ & 2 & 3 & 4 & 5 & 6 \\
\hline 2 & 1 & & & & \\
3 & 2 & 1 & & & \\
4 & 6 & 4 & 1 & & \\
5 & 24 & 18 & 8 & 1 & \\
6 & 120 & 96 & 58 & 13 & 1 \\
\multicolumn{5}{c}{$C_{\lambda}(n, k)$} & for $2 \leqslant n, k \leqslant 6$.
\end{tabular}

$\lambda=(2,2,2, \ldots)$

\begin{tabular}{c|ccccc}
$n \backslash k$ & 2 & 3 & 4 & 5 & 6 \\
\hline 2 & 1 & & & & \\
3 & 2 & 1 & & & \\
4 & 2 & 2 & 1 & & \\
5 & 4 & 14 & 8 & 1 & \\
6 & 4 & 32 & 38 & 12 & 1 \\
\multicolumn{5}{c}{$S_{\lambda}(n, k)$ for $2 \leqslant n, k \leqslant 6$}
\end{tabular}

\begin{tabular}{c|ccccc}
$n \backslash k$ & 2 & 3 & 4 & 5 & 6 \\
\hline 2 & 1 & & & & \\
3 & 2 & 1 & & & \\
4 & 6 & 4 & 1 & & \\
5 & 24 & 18 & 8 & 1 & \\
6 & 120 & 96 & 58 & 12 & 1 \\
\multicolumn{5}{c}{$C_{\lambda}(n, k)$} & for $2 \leqslant n, k \leqslant 6$.
\end{tabular}

$\lambda=(3,2,1, \ldots)$

\begin{tabular}{c|cccc}
$n \backslash k$ & 3 & 4 & 5 & 6 \\
\hline 3 & 1 & & & \\
4 & 3 & 1 & & \\
5 & 6 & 6 & 1 & \\
6 & 18 & 30 & 11 & 1
\end{tabular}

\begin{tabular}{c|cccc}
$n \backslash k$ & 3 & 4 & 5 & 6 \\
\hline 3 & 1 & & & \\
4 & 3 & 1 & & \\
5 & 12 & 6 & 1 & \\
6 & 60 & 36 & 11 & 1
\end{tabular}

$S_{\lambda}(n, k)$ for $3 \leqslant n, k \leqslant 6$ $C_{\lambda}(n, k)$ for $3 \leqslant n, k \leqslant 6$.

Table 2: Some tables for $S_{\lambda}(n, k), C_{\lambda}(n, k){ }^{1}$

\subsection{Main properties}

Let us define

$$
(x)_{0}:=1 ; \quad(x)_{m}:=x(x-1) \cdots(x-m+1) ; \quad(x)_{n}^{\lambda}:=(x)_{\lambda_{0}} \cdots(x)_{\lambda_{q_{n}}}(x)_{r_{n}} .
$$

Denote $\mathcal{D}_{\ell}:=x^{\ell} \partial^{\ell}$, then

$$
\mathcal{D}_{\ell_{1}} \mathcal{D}_{\ell_{2}}=\mathcal{D}_{\ell_{2}} \mathcal{D}_{\ell_{1}}
$$

or more precisely

$$
\mathcal{D}_{\ell_{1}} \mathcal{D}_{\ell_{2}}=\sum_{i} i !\left(\begin{array}{c}
\ell_{1} \\
i
\end{array}\right)\left(\begin{array}{c}
\ell_{2} \\
i
\end{array}\right) \mathcal{D}_{\ell_{1}+\ell_{2}-i}
$$

${ }^{1} S_{\lambda}(n, k)=C_{\lambda}(n, k)=0$ if $k<\lambda_{1}$ or $k>n$. If $\lambda=(1,1, \ldots)$ or $\lambda=(r, 1,1, \ldots)$ then the $\lambda$-Stirling numbers are referred to the ordinary Stirling numbers (A008275, A008277, A048994 in OEIS) or to the $r$-Stirling numbers (A143494, A143495, A143496, A193685, A143491, A143492, A143493 in OEIS [28]), respectively. Some values of $S_{\lambda}(n, k)$ (of second kind) are referred to A078739, A008297, A035342, A078740, A078741, A090214 in OEIS [28]. 
The last formula gives

$$
\mathcal{D}_{\ell}=\mathcal{D}_{\ell-1} \mathcal{D}_{1}-(\ell-1) \mathcal{D}_{\ell-1}
$$

Let $\mathcal{D}_{n}^{\lambda}$ be the operator defined as

$$
\mathcal{D}_{n}^{\lambda}:=\mathcal{D}_{\lambda_{0}} \cdots \mathcal{D}_{\lambda_{q}} \mathcal{D}_{r}
$$

Similar to equation (12) we can obtain that

$$
\mathcal{D}_{n}^{\lambda}=\mathcal{D}_{n-1}^{\lambda} \mathcal{D}_{1}-r_{n-1} \mathcal{D}_{n-1}^{\lambda}
$$

Theorem 6.3. The numbers $S_{\lambda}(n, k), C_{\lambda}(n, k)$ have the following properties.

(i) Recurrence relations

$$
S_{\lambda}(n, k)=S_{\lambda}(n-1, k-1)+\left(k-r_{n-1}\right) S_{\lambda}(n-1, k)
$$

with $S_{\lambda}(n, k)=0$ if $k<\lambda_{1}$ or $k>n ; S_{\lambda}(n, n)=1$.

$$
C_{\lambda}(n, k)=C_{\lambda}(n-1, k-1)+\left(n-1-r_{k}\right) C_{\lambda}(n-1, k)
$$

with $C_{\lambda}(n, k)=0$ if $k<\lambda_{1}$ or $k>n ; C_{\lambda}(n, n)=1$.

(ii) Expansions with differential operators

$$
\begin{gathered}
\mathcal{D}_{n}^{\lambda}=\sum_{k} S_{\lambda}(n, k) \mathcal{D}_{k}, \\
\mathcal{D}_{n}=\sum_{k}(-1)^{n-k} C_{\lambda}(n, k) \mathcal{D}_{k}^{\lambda} .
\end{gathered}
$$

(iii) Polynomial expansions

$$
\begin{gathered}
(x)_{n}^{\lambda}=\sum_{k=0}^{n} S_{\lambda}(n, k)(x)_{k}, \\
(x)_{n}=\sum_{k=0}^{n}(-1)^{n-k} C_{\lambda}(n, k)(x)_{k}^{\lambda}
\end{gathered}
$$

(iv) Orthogonality relations

$$
\begin{aligned}
& \sum_{k}(-1)^{k-m} S_{\lambda}(n, k) C_{\lambda}(k, m)=\delta_{n, m}, \\
& \sum_{k}(-1)^{k-m} C_{\lambda}(n, k) S_{\lambda}(k, m)=\delta_{n, m} .
\end{aligned}
$$

(v) Symmetric functions related formulas ${ }^{2}$

$$
S_{\lambda}(n, k)=\sum_{1 \leqslant i_{1} \leqslant \cdots \leqslant i_{n-k} \leqslant k} \prod_{j=1}^{n-k}\left(i_{j}-r_{i_{j}+j-1}\right),
$$

\footnotetext{
${ }^{2}$ These are related to complementary symmetric functions studied in [9].
} 


$$
C_{\lambda}(n, k)=\sum_{1 \leqslant i_{1}<\cdots<i_{n-k} \leqslant n} \prod_{j=1}^{n-k}\left(i_{j}-r_{i_{j}+1-j}\right) .
$$

(vi) The general formula for $S_{\lambda}(n, k)$ is given by

$$
S_{\lambda}(n, k)=\frac{1}{k !} \sum_{\ell=0}^{k}\left(\begin{array}{l}
k \\
\ell
\end{array}\right)(-1)^{k-\ell}(\ell)_{n}^{\lambda} .
$$

(vii) The following recurrence relations hold

$$
\begin{gathered}
S_{\lambda}(n, k)=\sum_{j=0}^{r_{n}}\left(\begin{array}{c}
r_{n} \\
j
\end{array}\right)(k-j)_{r_{n}-j} S_{\lambda}\left(n-r_{n}, k-j\right), \\
C_{\lambda}(n, k)=\sum_{j=r_{k}}^{n}\left(\begin{array}{c}
j \\
r_{k}
\end{array}\right)\left(n-r_{k}-1\right)_{j-r_{k}} C_{\lambda}\left(n-j, k-r_{k}\right) .
\end{gathered}
$$

(viii) The formulas

$$
\begin{gathered}
S_{\lambda}(n, k)=\sum_{j_{1}, \ldots, j_{q_{n}+1}}\left(\begin{array}{c}
r_{n} \\
j_{q_{n}+1}
\end{array}\right)\left(k-j_{q_{n}+1}\right)_{r_{n}-j_{q_{n}+1}} \prod_{\ell=1}^{q_{n}}\left(\begin{array}{c}
\lambda_{\ell} \\
j_{\ell}
\end{array}\right)\left(k-j_{\ell}-\cdots-j_{q_{n}+1}\right)_{\lambda_{\ell}-j_{\ell}} . \quad(27) \\
C_{\lambda}(n, k)=\sum_{j_{1}, \ldots, j_{q_{k}+1}}\left(\begin{array}{c}
j_{q_{k}+1} \\
r_{k}
\end{array}\right)\left(n-r_{k}-1\right)_{j_{q_{k}+1}-r_{k}} \prod_{\ell=1}^{q_{k}}\left(\begin{array}{c}
j_{\ell} \\
\lambda_{\ell}
\end{array}\right)\left(n-j_{\ell}-\cdots-j_{q_{k}+1}-r_{\ell}-1\right)_{j_{\ell}-r_{\ell}} .
\end{gathered}
$$

Proof. (i) Recurrence relations.

We can show that the number of described partitions has the same recurrence as (14). Note that the number of ways is 0 when $k<\lambda_{1}$ or $k>n$. If we consider the element $n$, then two cases are possible. If $n$ forms a separate block, then we have the number of ways to partition $[n-1]$ into $k-1$ parts over partition $\lambda$. If $n$ if placed in the block with some other elements except the restricted; this can be done in $\left(k-r_{n-1}\right)$ ways of any of partitions of $[n-1]$ into $k$ blocks over partitions $\lambda$. This argument implies the needed recurrence for $S_{\lambda}(n, k)$.

We show that the described number of ways satisfies the same recurrence as (15). Note that the number of ways is 0 when $k<\lambda_{1}$ or $k>n$. Consider the element $n$ and two cases. If $n$ form a singleton separate cycle, then the number of corresponding ways is the number of permutations of $[n-1]$ having $k-1$ cycles with the properties for $\lambda$ partition of $k-1$. If $n$ is in cycle with the other elements, then we can put $n$ in cycles after any element except last $r_{k}$ singletons. This gives $\left(n-1-r_{k}\right)$ ways for any permutation of $[n-1]$ with $k$ cycles and the described partition property. This argument clearly implies the needed recurrence for $C_{\lambda}(n, k)$. 
(ii) Expansions with differential operators.

The first relation

$$
\mathcal{D}_{n}^{\lambda}=\sum_{k=0}^{n} S_{\lambda}(n, k) \mathcal{D}_{k}
$$

implies from our general Theorem 4.1 on graph partitions.

From equation (12) we may easily obtain that

$$
\mathcal{D}_{n}^{\lambda}=\mathcal{D}_{n-1}^{\lambda} \mathcal{D}_{1}-r_{n-1} \mathcal{D}_{n-1}^{\lambda}
$$

For the companion expansion if for we know for $n-1$

$$
\mathcal{D}_{n-1}=\sum_{k=0}^{n-1}(-1)^{n-1-k} C_{\lambda}(n-1, k) \mathcal{D}_{k}^{\lambda},
$$

then for $n$ we get

$$
\begin{aligned}
\mathcal{D}_{n} & =\mathcal{D}_{n-1} \mathcal{D}_{1}-(n-1) \mathcal{D}_{n-1} \\
& =\sum_{k=0}^{n-1}(-1)^{n-1-k} C_{\lambda}(n-1, k) \mathcal{D}_{k}^{\lambda} \mathcal{D}_{1}-\sum_{k=0}^{n-1}(-1)^{n-1-k}(n-1) C_{\lambda}(n-1, k) \mathcal{D}_{k}^{\lambda} \\
& =\sum_{k=0}^{n-1}(-1)^{n-1-k} C_{\lambda}(n-1, k)\left(\mathcal{D}_{k+1}^{\lambda}+r_{k} \mathcal{D}_{k}^{\lambda}\right)-\sum_{k=0}^{n-1}(-1)^{n-1-k}(n-1) C_{\lambda}(n-1, k) \mathcal{D}_{k}^{\lambda} \\
& =\sum_{k=0}^{n}(-1)^{n-k}\left(C_{\lambda}(n-1, k-1)+\left(n-1-r_{k}\right) C_{\lambda}(n-1, k)\right) \mathcal{D}_{k}^{\lambda} \\
& =\sum_{k=0}^{n}(-1)^{n-k} C_{\lambda}(n, k) \mathcal{D}_{k}^{\lambda} .
\end{aligned}
$$

(iii) Polynomial expansions.

Applying the derivation operation to the function $x^{t}$ with a real parameter $t$, expansions (16), (17) yield

$$
\begin{gathered}
(t)_{\lambda_{0}} \cdots(t)_{\lambda_{q_{n}}}(t)_{r_{n}} x^{t}=\sum_{k=0}^{n} S_{\lambda}(n, k)(t)_{k} x^{t}, \\
(t)_{n} x^{t}=\sum_{k=0}^{n}(-1)^{n-k} C_{\lambda}(n, k)(t)_{\lambda_{0}} \cdots(t)_{\lambda_{q_{k}}}(t)_{r_{k}} x^{t} .
\end{gathered}
$$

The last two identities are polynomial relations in $t$ and hold for all $t$ which imply (18), (19).

(iv) Orthogonality relations directly imply from the inverse expansions (16), (17). 
(v) Symmetric function related formulas can be easily obtained by induction and use of the recurrence relations (14), (15).

(vi) The general formula for $S_{\lambda}(n, k)$. We will show that this formula holds using combinatorial interpretation of $S_{\lambda}(n, k)$ and the inclusion-exclusion principle. Suppose that blocks are ordered. Let us enumerate them as $1, \ldots, k$.

Denote by $A_{i}(1 \leqslant i \leqslant k)$ the set of corresponding restricted (up to $\lambda$ ) arrangements of $[n]$ into $k$ ordered blocks such that the $i$-th block is empty. Let $A$ be the number of all restricted arrangements of $[n]$ into $k$ blocks (some of them might be empty). Then it is clear that

$$
k ! S_{\lambda}(n, k)=A-\left|A_{1} \cup \cdots \cup A_{k}\right| .
$$

Note that

$$
A=(k)_{\lambda_{1}} \ldots(k)_{\lambda_{q_{n}}}(k)_{r_{n}}
$$

and

$$
\left|A_{i_{1}} \cap \cdots \cap A_{i_{k-\ell}}\right|=(\ell)_{\lambda_{1}} \ldots(\ell)_{\lambda_{q_{n}}}(\ell)_{r_{n}}
$$

for any set of indices $1 \leqslant i_{1}<\cdots<i_{k-\ell} \leqslant k$. Therefore, using the inclusion-exclusion principle we get

$$
\begin{aligned}
k ! S_{\lambda}(n, k) & =A-\left|A_{1} \cup \cdots \cup A_{k}\right| \\
& =(k)_{\lambda_{1}} \ldots(k)_{\lambda_{q_{n}}}(k)_{r_{n}}-\sum_{\ell=0}^{k-1} \sum_{1 \leqslant i_{1}<\cdots<i_{k-\ell} \leqslant k}(-1)^{k-1-\ell}\left|A_{i_{1}} \cap \cdots \cap A_{i_{k-\ell}}\right| \\
& =(k)_{\lambda_{1}} \ldots(k)_{\lambda_{q_{n}}}(k)_{r_{n}}-\sum_{\ell=0}^{k-1}(-1)^{k-1-\ell}\left(\begin{array}{c}
k \\
\ell
\end{array}\right)(\ell)_{\lambda_{1}} \ldots(\ell)_{\lambda_{q_{n}}}(\ell)_{r_{n}} \\
& =\sum_{\ell=0}^{k}(-1)^{k-\ell}\left(\begin{array}{c}
k \\
\ell
\end{array}\right)(\ell)_{\lambda_{1}} \ldots(\ell)_{\lambda_{q_{n}}}(\ell)_{r_{n}} .
\end{aligned}
$$

(vii) Recurrence relations.

Recurrence (25). Suppose that $j$ elements of the last $r_{n}$ in $[n]$ are singleton blocks. We can choose these elements in $\left(\begin{array}{c}r_{n} \\ j\end{array}\right)$ ways. The remaining $\left(r_{n}-j\right)$ elements should be put in distinct $(k-j)$ blocks of any of $S_{\lambda}\left(n-r_{n}, k-j\right)$ partitions, which can be done $(k-j)_{r_{n}-j}$ times.

Recurrence (26). We may choose the needed $r_{k}$ singleton cycles from the last $j$ elements of $[n]$. This can be done in $\left(\begin{array}{c}j \\ r_{k}\end{array}\right)$ ways. The remaining $\left(j-r_{k}\right)$ elements should be put in the first $(n-j)$ cycles of any of $C_{\lambda}\left(n-j, k-r_{k}\right)$ permutations, which can be done $\left(n-r_{k}-1\right)_{j-r_{k}}$ times.

(viii) The last formulas (27), (28) imply from iterative use of relations (25), (26).

\section{References}

[1] B. R. Alspach and N. J. Pullman. Path decompositions of digraphs. Bull. Austral. Math. Soc., 10:421-427, 1974. 
[2] P. Balister. Packing digraphs with directed closed trails. Combin. Probab. Comput., 121-15, 2003.

[3] P. Blasiak and P. Flajolet. Combinatorial models of creation-annihilation. Séminaire Lotharingien de Combinatoire, 65:Article B65c, 2011.

[4] P. Blasiak, K. A. Penson, and A. I. Solomon. The boson normal ordering problem and generalized Bell numbers. Ann. Comb., 7:127-139, 2003.

[5] A. Z. Broder. The $r$-Stirling numbers. Discrete Math., 49:241-259, 1984.

[6] W. Chen, E. Deng, and R. Du. Reduction of $m$-regular noncrossing partitions. European J. Combin., 26:237-243, 2005.

[7] P. Codara, O. M. D'Antona, and P. Hell. A simple combinatorial interpretation of certain generalized Bell and Stirling numbers. Discrete Math., 318:53-57, 2014.

[8] F. R. K. Chung and R. L. Graham. Recent results in graph decompositions. Combinatorics (HNV Temperley, ed.), London Math. Soc., Lecture Notes Series, 52:103$124,1981$.

[9] E. Damiani, O. M. D'Antona, and D. E. Loeb. The complementary symmetric functions: Connection constants using negative sets. Adv. Math., 135:207-219, 1998.

[10] O. M. D'Antona and E. Munarini. A combinatorial interpretation of the connection constants for persistent sequences of polynomials. European J. Combin., 26:11051118, 2005.

[11] B. S. El-Desouky. The multiparameter non-central Stirling numbers. Fibonacci Quart, 32:218-225, 1994.

[12] B. S. El-Desouky, N. P. Cakic, and T. Mansour. Modified approach to generalized Stirling numbers via differential operators. Appl. Math. Lett., 23:115-120, 2010.

[13] A. Donald. An upper bound for the path number of a graph. J. Graph Theory, 4:189-201, 1980.

[14] B. Duncan and R. Peele. Bell and Stirling numbers for graphs. J. Integer Seq. 12:Article 09.7.1, 2012

[15] A. Dzhumadil'daev and D. Yeliussizov. Path decompositions of digraphs and their applications to Weyl algebra. Adv. in Appl. Math., 67:36-54, 2015.

[16] G. Fan. Path decompositions and Gallai's conjecture. J. Combin. Theory Ser. B, 93:117-125, 2003.

[17] P. Harding and S. McGuinness. Gallai's Conjecture For Graphs of Girth at Least Four. J. Graph Theory, 75:256-274, 2014.

[18] J. Katriel and M. Kibler. Normal ordering for deformed boson operators and operator-valued deformed Stirling numbers. Journal of Physics A: Mathematical and General, 25:2683, 1992.

[19] L. Lovász. On covering of graphs. in Theory of Graphs, Proc. Coll. Tihany, 1966. 
[20] T. Mansour, M. Schork, and M. Shattuck. On a new family of generalized Stirling and Bell numbers. Electron. J. Combin., 18:\#P77, 2011.

[21] T. Mansour, M. Schork and M. Shattuck. The generalized Stirling and Bell numbers revisited. J. Integer Seq., 15:Article 12.8.3, 2012.

[22] T. Mansour and M. Schork. Commutation Relations, Normal Ordering, and Stirling Numbers. CRC Press, 2015.

[23] C. J. H. McDiarmid. Path-partition structures of graphs and digraphs. Proc. Lond. Math. Soc., 3.4:750-767, 1974.

[24] M. A. Méndez, P. Blasiak, and K. A. Penson. Combinatorial approach to generalized Bell and Stirling numbers and boson normal ordering problem. J. Math. Phys., 46:083511-1-8, 2005.

[25] M. Mihoubi and M.S. Maamra. The $\left(r_{1}, \ldots, r_{p}\right)$-Stirling numbers of the second kind. Integers, 12:\#A35, 2012.

[26] G. Olshanski. Representations of infinite-dimensional classical groups, limits of enveloping algebras, and Yangians. Topics in representation theory, Advances in Soviet Mathematics (A. Kirillov, ed.), vol. 2, AMS, Providence, RI, 1-66, 1991.

[27] A. Okounkov. Young basis, Wick formula, and higher Capelli identities. Int. Math. Res. Not., 1996.17: 817-839, 1996.

[28] The Online Encyclopedia of Integer Sequences (OEIS), http://oeis .org

[29] R. Stanley. Enumerative Combinatorics, Vol. 1. Cambridge University Press, Cambridge, 2011.

[30] A. Varvak. Rook numbers and the normal ordering problem. J. Combin. Theory Ser. A, 112:292-307, 2005. 